\title{
Students' Satisfaction with Simulation Learning Environment in Relation to Self-confidence and Learning Achievement
}

\author{
Abdulaziz Saleh Aljohani ${ }^{1}$, Qayomi Karim ${ }^{2}$ and Pachev George ${ }^{3}$ \\ 1. Department of Family Medicine, Security Forces Medical Services, Riyadh, Kingdome of Saudi Arabia \\ 2. Center of Excellence for Simulation Education and Innovation (CESEI), University of British Columbia, Canada \\ 3. Department of Medical Education, University of British Columbia, Canada
}

\begin{abstract}
The evolution in patient simulation as educational tool is being driven by a number of factors. Priority of patient safety, patient availability, and the ever increasing body of medical knowledge presents new challenges to curriculum planners. Student's satisfaction is an important element of the investigation of simulation learning environment efficacy. There are suggestions that student satisfaction may have some correlation with self-confidence and learning achievement. This is a prospective exploratory study that evaluates learners' satisfaction with simulation learning environment and self-conference utilized satisfaction with SSE (simulation experience scale) and pre and post simulation test for learning achievement. Participants was third-year medical students $(\mathrm{n}=45)$ participating in their regular simulation-based session at Center of Excellence for Simulation Education and Innovation (CESEI), University of British Columbia. A comparison between pre- and post-test results was conducted on the basis of $t$-test for related samples. Correlation was used to explore the relationships among students satisfaction with the simulation environment and students' self-confidence and achievement. The simulation exercise, completed by 45 students, increased correct test answers on average from $72 \%$ to $89 \%$ ( $P$-Value $<0.0001$ by paired $t$-test). Increases in test results were between pre- and post- simulation identical multiple choice questions. The mean score for satisfaction with simulation environment items was 4.47, SD (0.45), using a 5-point Likert scale with 5 = strongly agree, $4=$ agree, $3=$ neutral, $2=$ disagree, and $1=$ strongly disagree. The mean score for self-confidence in performing BLS, evaluating and managing acute cardiac patient was 3.83, SD (1.02). For evaluating the relationship between students satisfaction with simulation learning environment and learning achievement, bivariate analysis revealed a significant positive relationship between satisfaction with simulation learning environment and learning achievement (Pearson $\mathrm{r}=0.80, P$-Value $<0.01$ ). The magnitude or strength of the correlation coefficient $(\mathrm{r}=0.80)$ indicated satisfaction with simulation environment and learning achievement have a strong effect and positive correlation. Simulation learning environment for medical students is effective in improving students' overall comprehension and better learning achievements. Furthermore, students' basic clinical skills are improved associated with higher self-confidence.
\end{abstract}

Key words: Simulation, self-confidence, learning achievement.

\section{Introduction}

\subsection{Importance of Clinical Environment in Medical} Education

The clinical learning environment is irreplaceable in preparing students for their professional role [1]. It is increasingly being recognized that these learning

Corresponding author: Abdulaziz Saleh Aljohani, professor, research fields: family medicine, palliative care and medical education. experiences are influenced by the quality of the clinical placement and supported facilitation [2]. In the clinical learning environment, there is no guarantee that a student will be exposed to the variety of patients and conditions necessary for learning the appropriate clinical skills. Moreover, currently most clinical learning environments involve students as passive observers, to the detriment of their learning. Simulation offers an alternative learning environment allowing for a wide range of skills to be practiced and mastered. 
Specific learning goals and objectives can be defined, and all learners can successfully fulfill the goals and objectives, because learning takes place using trained instructors in dedicating teaching time rather than patient-care time.

\subsection{Technology Development and Simulation}

Technology use in the medical education process has, in many ways, kept pace with the exponential growth in its use across all facets of life. One relatively recent technology in the health professions education is the use of patient simulators of varying degrees of fidelity. These devices replicate many human anatomical features and physiological functions. Learners interact with the simulator, discovering critical assessment information in the same manner they would with real patients. Once the simulator's medical condition is identified, learners then proceed with treatment options in an effort to correct the simulator's condition. The patient simulator experience combined with clinical practice experiences can provide students an appropriate background for their future career.

\subsection{Factors for Interest in Simulation}

The evolution in patient simulation as educational tool is being driven by a number of factors. Priority of patient safety, patient availability, and the ever increasing body of medical knowledge presents new challenges to curriculum planners. Additionally, simulation offers the capability to create standardization in evaluation by providing consistent replication of patient cases to improved learner outcome measurements.

\subsection{What Simulation is Needed? (High-fidelity, Educational Principles)}

The simulation learning environment should provide the real-life context that is essential for the development of the knowledge, values and skills required of a medical student. The simulation should provide access to a suitable environment, containing the tools, instruments or resources required to gain an understanding of domain concepts and allow the student to become aware of incompleteness and inconsistency in current knowledge. The current need for training evaluation extends beyond the well-established validation of simulators as training tools. Effective evaluation structures increase the effective use of training systems and the application of skills acquired during the training program, further enabling the scientific validation of all facets of the training process. Evaluation of simulation learning environment using student's level of satisfaction, improvements in self-confidence and learning achievement represents the ability to understand the multifaceted training process and the learning outcomes produced, allowing application of this knowledge for further advancement of simulation use and effective training techniques.

\subsection{Satisfaction}

The quality of medical education, was indicated by students' satisfaction with clinical practice and the simulation learning environment as a whole. Student satisfaction is important element in the investigation the efficacy of simulation learning environment. Student satisfaction is important for engaged and meaningful learning and it facilitates active and purposeful participation in the simulation experiences [3]. Student satisfaction is the subjective perceptions, on students' part, of how well a simulation learning environment supports their learning. Strong student satisfaction implies that appropriately challenging instructional methods are serving to trigger students' thinking and learning. There are also suggestions that student satisfaction may have some correlation with performance [4]. Students need to be confident that they can be successful in the simulation learning environment. Few studies have explored in a rigorous relation between student satisfaction with simulation learning environment and student confidence and achievement. 


\subsection{Studies on Satisfaction}

A number of studies have reported on the levels of satisfaction as being very positive after simulation-based education programs [5-8]. Kardong-Edgren et al. [9] examined student satisfaction in first-year students exposed to three simulation sessions using medium fidelity HPSMs and high levels of student satisfaction were reported. Previous studies show that students' academic success relies on certain features of learning environments, notably on small-group work and problem-solving exercises [10-12]. Students can encounter difficulties in simulation learning environment [13, 14]. They reported that some students concentrate on manipulating the software to complete tasks, without gaining a deeper understanding of the abstract principles that govern simulation behavior.

\subsection{Studies on Confidence and Achievement}

Confidence is vital to the clinician taking action. As Maibach, Schieber, and Carroll [15] noted, even clinicians with adequate knowledge and skills may be reluctant to take appropriate action unless they are confident in their abilities. In reviewing the literature, learner feelings of self-confidence tend to be improved when the learning experience is simulation-based. Euliano [16] reviewed the results of student evaluations of a simulation based course that also utilized problem-based learning techniques. Using a pretest/posttest design, learner confidence in their knowledge of the material significantly improved after participation in the simulation program $(P$-Value $<$ 0.0001). Henrichs, Rule, Grady, and Ellis [17] performed a qualitative study of 12 first year nurse anesthesia students to determine their perceptions about simulation as a learning strategy. Their results identified that simulation increased student's confidence level. Wayne et al. [18] conducted a follow-up survey with 40 second year internal medicine residents after having completed an ACLS-like simulation-based education program. They reported that the subjects were uniformly positive about the ability of the simulation-based education experience to increase their clinical capabilities and improve their confidence to respond to ACLS emergencies in the clinical setting. Both satisfaction and self-confidence are subjective opinions and can over substantial variability. The interpretation of these opinions needs to be anchored by more "objective" constructs like learning achievement.

For continuing education and practice to enhance student achievement, Dalley, Robinson, Weller, and Caldwell [19] conducted a randomized static-group comparison study of 18 anesthesiology students receiving training on a new anesthesia delivery system. One group received the standard didactic training on the device. The other group received that same training augmented with practical experience in the use of the device with a patient simulator. After training, both groups reported a high degree of confidence in their ability to use the device $(P$-Value $=0.203)$. However, when examined in a practical posttest, the group whose training employed the simulator significantly outperformed the control group in two simulation scenarios in which device complications were introduced $(P$-Value $=0.0113$ and $P$-Value $=0.0413)$.

Wyatt, Fallows, and Archer [20] conducted a randomized pretest/posttest control group study that examined the error rate in paramedics comparing simulation-based education with case-study based teaching. Their results showed simulation-based education had a significantly improved outcome greater than that of the case-study based teaching group $(P$-Value $=0.008)$.

Shapiro et al. [21] conducted a pretest/posttest study that compared the impact of an emergency department team training course that included an 8-hour simulator session against another group that completed the same training but spent an 8-hour shift in the emergency department. Following the intervention, each group was observed and scored on team behavior. Comparisons between pretest and posttest scores on the 
level of team behavior showed the simulation group had improved, although the level did not reach significance $(P$-Value $=0.07)$, while the group that completed a regular 8-hour shift showed no gains at all $(P$-Value $=0.55)$.

The purpose of this study is to explore the relation between student satisfaction with simulation learning environment and their self-confidence and learning achievement. We hypothesised that satisfaction with simulation learning environment will be related to student confidence and learning achievement.

\section{Methods}

\subsection{Study Design}

This is a prospective exploratory study that evaluates learner satisfaction with simulation learning environment and self-conference utilizing satisfaction with simulation experience (SSE) scale and pre and post simulation test for learning achievement.

The study will be conducted in Canada, in Vancouver General Hospital at the Center of Excellence for Simulation Education and Innovation (CESEI). The Center offers different types of simulation sessions for medical students, physicians, nurses and allied health professionals.

\subsection{Setting and Participants}

Study participants will include third-year medical students participating in their regular simulation-based session at CESEI, University of British Columbia. Students' consent to participate will be sought before the session by the principal investigator (who is not in a position of power relative to the participants).

\subsection{Measures}

For satisfaction with simulation learning environment and self-confidence:

The 18-item satisfaction with simulation experience (SSE) scale, developed by Levett-Jones et al. [22] uses a 5-point Likert scale (1 Strongly Disagree, 2 Disagree, 3 Unsure, 4 Agree and 5 Strongly Agree) to determine the extent the participants agree with a set of statements. The instrument has three subscales: debrief and reflection $(n=9)$, clinical reasoning $(n=5)$ and clinical learning $(\mathrm{n}=4)$. The SSE was psychometrically tested. The debrief and reflection subscale contained items such as "The facilitator summarized important issues during the debriefing". The clinical reasoning is assessed with items such as "The simulation developed my clinical reasoning skills". The clinical learning is assessed with items such as "The simulation tested my clinical ability". The SSE scale was adapted to include two more subscales to cover two additional aspects of simulations: simulation environment $(n=6)$ and self-confidence $(n=5)$. These two subscales were developed after reviewing different types of scales that used in different studies with peer-review process.

The simulation environment subscales include important aspects supporting a positive learning environment: creating an atmosphere in which the ability of individuals to function professionally is optimized such as reality to clinical practice; equipment and physical aspects of the learning environment.

The fidelity of the simulation environment will be assessed with items such as "the patient simulator space resembled a real clinical care setting".

The self-confidence subscale includes items related to one's confidence with ability to make appropriate patient assessments, make decisions about appropriate interventions, evaluate the effectiveness of interventions, and confidence in managing a semiconscious patient.

The self-confidence will be assessed with items such as "I gained confidence in term of my ability to make decisions about appropriate interventions".

\subsection{For Learning Achievement}

The participants will complete pre and post-tests containing 20 items. The multiple-choice examination questions will obtained from a variety of sources, 
including multiple textbook test banks, and were used to evaluate cognitive knowledge of the students based on the objectives of the simulation session. Several faculty members will review the 20-item exams for content validity to determine whether the questions measured the session content.

\subsection{Simulation and Procedure}

The simulation scenario, acute coronary syndrome (ACS), will be programmed into computerized simulation mannequin (Laerdal SimMan3G) with ability to simulate adverse physiologic response. For example, a decrease in oxygen saturation would occur if bag-valve mask ventilation was not started within 5 min, or an increase in blood pressure would occur if the medical students give intravenous fluids.

The simulation exercise for the current study, will be piloted with three third-year medical students, revised with input from two attending physicians, and then finalized. Two week before the scheduled simulation exercise, participants will review on-line didactic materials followed by interactive video-teaching tools and practice skills during the hands-on session in the Sim-Lab.

Once students arrive for their scheduled simulation session, the principal investigator will meet them in the main lecture theater of the Center and the research project will be explained. The principal investigator will then invite students to participate. An information statement well be provided to the participating students and they will be asked to sign a consent form prior to participating. Prior to implementation, the research project will be explained also to the instructors who will observe the students during the simulation session. The instructors will be asked to complete a performance checklist and provide feedback immediately following each session.

For students' learning achievement, the students complete an online pre-test immediately before starting the exercise. The pre-test consist of 20 multiple-choice questions testing the students' understanding. The knowable content of the pre-test based on the objectives of the simulation session. After the simulation exercise and a constructive feedback session, the students will complete a post-test identical to the pre-test.

After completing the pretest and before the actual simulation exercise, the students will spend some time with a clinical instructor becoming familiar with the mannequin and the laboratory layout and equipment. Details about the setting, the available resources, and the tasks at hand will be explained.

Then, students will be given 20 min to complete a simulation exercise using the high-fidelity mannequin (Laerdal SimMan3G). A brief history will provide on the computer screen featuring history of present illness, past medical history, medication history and basic vital signs. The instructor will give additional history if requested by the students and also give a few prompts if the students needed assistance. Patient's vital signs will displayed continuously on patient monitor and adjusted according to the clinical situation.

Students will instruct to collaborate with each other in the clinical care of the patient, including history tacking at the bedside, physical examination, monitoring of vital signs, generation of a differential diagnosis, and initiation of workup and therapy. The mannequin will be programmed to react to student interventions.

After the simulation exercise, the students will be debriefed by an attending instructor. Immediately after the debriefing, the students will complete a post-test which will be identical to the pretest.

\subsection{Data Analysis}

Descriptive and correlational methods will be use to describe the relationships between variables use in the study. Descriptive statistics will be used to summarize satisfaction variables (debrief and reflection, clinical reasoning, clinical learning and simulation environment). Through the use of charts and tables 
these variables provide a pictorial representation of the relationships will observe.

A bivariate correlational analysis utilizing the Pearson method will be used to determine if there any relationships between students satisfaction with simulation learning environment and self-confidence and learning achievement. The Pearson coefficient from these analyses enables the researcher to determine the degree, or strength, of the relationships.

\section{Result}

The simulation exercise, completed by 45 students, increased correct test answers on average from $72 \%$ to $89 \%$ ( $P$-Value $<0.0001$ by paired $t$-test $)$. Increases in test results were between pre- and post- simulation identical multiple choice questions. The simulation based education had a highly significant advantage in performance in the posttest $(P$-Value $<0.0001)$. The results showed that after the simulation intervention, there was a great improvement in the motivation and academic achievement of the students.

The mean score for satisfaction with simulation environment items was 4.47, SD (0.45), using a 5-point Likert scale with $5=$ strongly agree, $4=$ agree, $3=$ neutral, 2 = disagree, and $1=$ strongly disagree. Results showed a very positive agreement that indicated a high degree of satisfaction with the simulation session. During debriefings associated with the scenarios, validity of the program was established as there was uniform agreement that the errors highlighted in the simulation session were errors that were commonly seen in practice. The students felt the simulation was realistic, represented the learning objectives, was a valuable learning experience, and helped link theoretical aspects of care to practical applications. On individual items related to knowledge gain, the subjects rated the simulation highly with a mean range of responses being 4.6 to 4.73 .

The mean score for self-confidence in performing BLS, evaluating and managing acute cardiac patient was 3.83, SD (1.02). Results showed students' confidence in their ability to provide patient care after simulation based educational session have been positive. As this study was performed with third-year students, some of negative factors were reported such as anxiety and fixation errors may correlate to their experience level with clinical practice in general. Experience plays a significant role in the development of healthcare provider confidence. Confidence is vital to the clinician taking action, even clinicians with adequate knowledge and skills may be reluctant to take appropriate action unless they are confident in their abilities.

For evaluating the relationship between students satisfaction with simulation learning environment and learning achievement, bivariate analysis revealed a significant positive relationship between satisfaction with simulation learning environment and learning achievement (Pearson $\mathrm{r}=0.80, P$-Value $<0.01$ ). The magnitude or strength of the correlation coefficient $(\mathrm{r}=$ 0.80 ) indicated satisfaction with simulation environment and learning achievement have a strong effect and positive correlation.

\section{Discussion}

Students' overall satisfaction with the simulation learning environment was found to be positive in this study. This finding may be explained by the possibility students were actively involved in the learning experience, were given ample opportunity to practice comfort care measures, ask questions, and receive feedback from their instructor.

Most students in this study indicated that their confidence in their skills increased after the simulation activity when: first, simulation training was provided in well occupied simulation environment as an alternate clinical experience; second, when performing procedures; third, after participating in a workshop on cardiovascular physiology; and fourth, when using a high-fidelity simulator.

This study demonstrates a positive relationship between self-confidence, achievement and satisfaction 
in learning. Skills laboratory and simulation centers faculty should continually aim to build and evaluate student self-confidence and satisfaction with learning by fostering an environment that challenges students in a respectful manner.

\section{Conclusions}

Overall, these students found the experience enhanced their learning and helped improve their decision making skills. On the whole, respondents were very positive regarding the transferability, realism and value of the patient simulation experience.

Results from this study support the use of simulation learning environment with medical students as well as the need to provide a multitude of interactive learning methods that challenge students at their current competency level.

Faculties are encouraged to consider simulation as a learning strategy for medical students learning effective comfort care measures. With technological advances in health care, it is even more important that faculty incorporate diverse learning methods to optimize students' adaptability to an ever-changing, complex clinical environment. In the end, faculty want students to be satisfied with their learning, but even more important, they want students to be effective with their patients.

\section{References}

[1] Dunn, S. V., and Hansford, B. 1997. "Undergraduate Nursing Students' Perceptions of Their Clinical Learning Environment." Journal of Advanced Nursing 25 (6): 1299-306.

[2] Sand-Jecklin, K. 2009. “Assessing Nursing Student Perceptions of the Clinical Learning Enviroment: Refinement and Testing of the SECEE Inventory." Journal of Nursing Measurement 17 (3): 232-46.

[3] Prion, S. 2008. "A Practical Framework for Evaluating the Impact of Clinical Simulation Experiences in Prelicensure Nursing Education." (Electronic version). Clinical Simulation in Nursing 4 (5): e69-78.

[4] Bremner, M., Aduddell, K., Bennett, F., and Van Geest, J. 2006. "The Use of Human Patient Simulators: Best Practice with Novice Nursing Students." Nurse Educator 31 (4): 170-4.
[5] Block, E. F., Lottenberg, L., Flint, L., Jakobsen, J., and Liebnitzky, D. 2002. "Use of a Human Patient Simulator for the Advanced Trauma Life Support Course." American Surgeon 68 (7): 648-51.

[6] Cleave-Hogg, D., and Morgan, P. J. 2002. "Experiential Learning in an Anaesthesia Simulation Centre: Analysis of Students' Comments." Medical Teacher 24 (1): 23-6.

[7] Morgan, P. J., Cleave-Hogg, D., McIlroy, J., and Devitt, J. H. 2002. "Simulation Technology: A Comparison of Experiential and Visual Learning for Undergraduate Medical Students." Anesthesiology 96 (1): 10-6.

[8] von Lubitz, D. K., Carrasco, B., Gabbrielli, F., Ludwig, T., Levine, H., and Patricelli, F., et al. 2003. "Transatlantic Medical Education: Preliminary Data on Distance-based High-fidelity Human Patient Simulation Training." Studies in Health Technology and Informatics 94: 379-85.

[9] Kardong-Edgren, S., Starkweather, A., and Ward, L. 2008. "The Integration of Simulation into a Clinical Foundation of Nursing Course: Student and Faculty Perception." International Journal of Nursing Education Scholarship 5 (1). doi:10.2202/1548-923X.1603 (Article 26).

[10] Gokhale, A. A. 1995. "Collaborative Learning Enhances Critical Thinking." Journal of Technology Education 7 (1).

[11] Johnson, D. W., Johnson, R. T., and Smith, K. 2007. “The State of Cooperative Learning in Postsecondary and Professional Settings." Educational Psychology Review 19: 15-29.

[12] Jones, P. R. 2006. "Using Groups in Criminal Justice Courses: Some New Twists on a Traditional Pedagogical Tool." Journal of Criminal Justice Education 17 (1): $87-101$

[13] Goodyear, P., and Taik, K. 1989. "Learning with Computer-based Simulations: Tutoring and Student Modeling Requirements for an Intelligent Learning Advisor." Proceedings of the EARLI Conference, Madrid, Spain.

[14] Laurillard, D. 1992. "Learning through Collaborative Computer Simulations." British Journal of Educational Technology 23: 164-71.

[15] Maibach, E. W., Schieber, R. A., and Carroll, M. F. 1996. "Self-efficacy in Pediatric Resuscitation: Implications for Education and Performance." Pediatrics 97: 94-9.

[16] Euliano, T. 2001. "Small Group Teaching: Clinical Correlation with a Human Patient Simulator." Advances in Physiology Education 25 (1-4): 36-43.

[17] Henrichs, B., Rule, A., Grady, M., and Ellis, W. 2002. "Nurse Anesthesia Students' Perceptions of the Anesthesia Patient Simulator: A Qualitative Study." AANA Journal 70 (3): 219-25.

[18] Wayne, D. B., Butter, J., Siddal, V. J., Fudala, M. J., Wade, L. D., and Feinglass, J., et al. 2006. "Mastery Learning of 

Self-confidence and Learning Achievement

Advanced Cardiac Life Support Skills by Internal Medicine Residents using Simulation Technology and Deliberate Practice." Journal of General Internal Medicine 21 (2): 251.

[19] Dalley, P., Robinson, B., Weller, J., and Caldwell, C. 2004. "The Use of High-fidelity Human Patient Simulation and the Introduction of New Anesthesia Delivery Systems." Anesthesia and Analgesia 99 (6): 1737-41.

[20] Wyatt, A., Fallows, B., and Archer, F. 2004. "Do Clinical Simulations using a Human Patient Simulator in the Education of Paramedics in Trauma Care Reduce Error Rates in Preclinical Performance [Abstract]?” Prehospital
Emergency Care 8 (4): 435-6.

[21] Shapiro, M. J., Morey, J. C., Small, S. D., Langford, V., Kaylor, C. J., and Jagminas, L., et al. 2004. "Simulation Based Teamwork Training for Emergency Department Staff: Does it Improve Clinical Team Performance when added to an Existing Didactic Teamwork Curriculum?" Quality and Safety in Health Care 13 (6): 417-21.

[22] Tracy, L.-J., Michelle, M., Samuel, L., Danielle, N., Kerry, H., Jennifer, D., Carol, A., and Jan, R. 2011. "The Development and Psychometric Testing of the Satisfaction with Simulation Experience Scale." Nurse Education Today 31 (7): 705-10. 